\title{
LETTER
}

\section{Recombinant factor VIla and factor VIII treatment for acquired factor VIII deficiency: a case of repeated thrombotic endotracheal occlusion in a mechanically ventilated patient}

\author{
Stefan Lauer*1, Martin Westphal',2, Lars G Fischer', Andreas Meißner', Hugo Van Aken' and Hendrik Freise'
}

Acquired hemophilia A is caused by autoantibodies to coagulation factor VIII (FVIII). Preoperative diagnosis is impaired by the lack of a personal or family history of bleeding episodes. Therefore, surgical procedures can lead to life-threatening hemorrhage [1].

We present a case of a 72-year-old woman who had no history of coagulopathy and who was undergoing hysteroscopy and abrasion in a district hospital. Persistent vaginal bleeding began immediately after surgery, resulting in vaginal hysterectomy on postoperative day (POD) 7 and multiple re-laparotomies with intermittent abdominal packing between PODs 8 and 37. Acquired FVIII deficiency was suspected on POD 30, but a disseminated intravascular coagulation and treatment with various pro-coagulants made the definitive diagnosis difficult. Bolus applications of recombinant factor VIIa (rFVIIa) and von Willebrandt factor-FVIII complex between PODs 33 and 38 plus prednisolone pulse therapy remained ineffective. Acquired FVIII deficiency was finally diagnosed upon admission of the intubated and ventilated patient to the intensive care unit of a university hospital on POD 40. Further FVIII was administered because of persistent surgical bleeding. Following massive abdominal bleeding on POD 44, the patient received FVIII for the next 24 hours and rFVIIa until POD 49. On POD 47, the abdominal bleeding ceased.

Three life-threatening airway complications occurred under rFVIIa and FVIII therapy. On POD 40, the endotracheal tube (ETT) was completely obstructed by blood clots. On POD 46, pulmonary gas exchange rapidly deteriorated because of a huge thrombus in the left main bronchus $(2 \times 5 \mathrm{~cm})$. On POD 49, a large endobronchial

\footnotetext{
*Correspondence: stlauer@gmx.net

'Department of Anesthesiology and Intensive Care Medicine, University Hospital

Muenster, Albert-Schweitzer-Str. 33, 48149 Muenster, Germany

Full list of author information is available at the end of the article
}

thrombus $(2 \times 7 \mathrm{~cm})$, which was attached to the tip of the ETT, was fortunately extracted during the emergency removal of the ETT (Figure 1a,b).

Each time, bronchoscopy showed only moderate diffuse bleeding from minor mucosal erosions. After the critical incident on POD 49, the patient was weaned from the respirator and eventually fully recovered.

Intravascular thromboembolic events under treatment with rFVIIa and FVIII present a well-known complication $[2,3]$. Life-threatening thrombotic airway obstructions in a mechanically ventilated patient, however, are a rare complication. The fact that activated partial thromboplastin times (aPTTs) were spontaneously prolonged (53 to 66 seconds) and active bleeding was present during these events highlights the unpredictable local imbalance of pro- and anticoagulant effects during such a treatment. In conclusion, intensive care clinicians should be aware that minor lacerations of the bronchial mucosa in mechanically ventilated patients could lead to blood clots and critical airway obstruction under the treatment with rFVIIa and FVIII.

\section{Abbreviations \\ ETT, endotracheal tube; FVIII, factor VIII; POD, postoperative day; rFVIIa, recombinant factor VIla.}

\section{Competing interests}

The authors declare that they have no competing interests.

\section{Acknowledgements}

Written consent for publication was obtained from the patient.

\section{Author details}

'Department of Anesthesiology and Intensive Care Medicine, University Hospital Muenster, Albert-Schweitzer-Str. 33, 48149 Muenster, Germany. ${ }^{2}$ Fresenius Kabi AG, Else-Kröner-Strasse 1, 61352 Bad Homburg, Germany.

\section{Published: 9 March 2011}

\footnotetext{
References

1. Brack A, Vogeler S, Hilpert J, Berger G, Buhr HJ, Koscielny J: Acquired factor VIII inhibitor. Anesthesiology 2009, 111:1151-1154.

2. Barletta JF, Cooper B, Ohlinger MJ: Adverse drug events associated with disorders of coagulation. Crit Care Med 2010, 38:S198-218.

3. Vincent JL, Rossaint R, Riou B, Ozier Y, Zideman D, Spahn DR:
} 

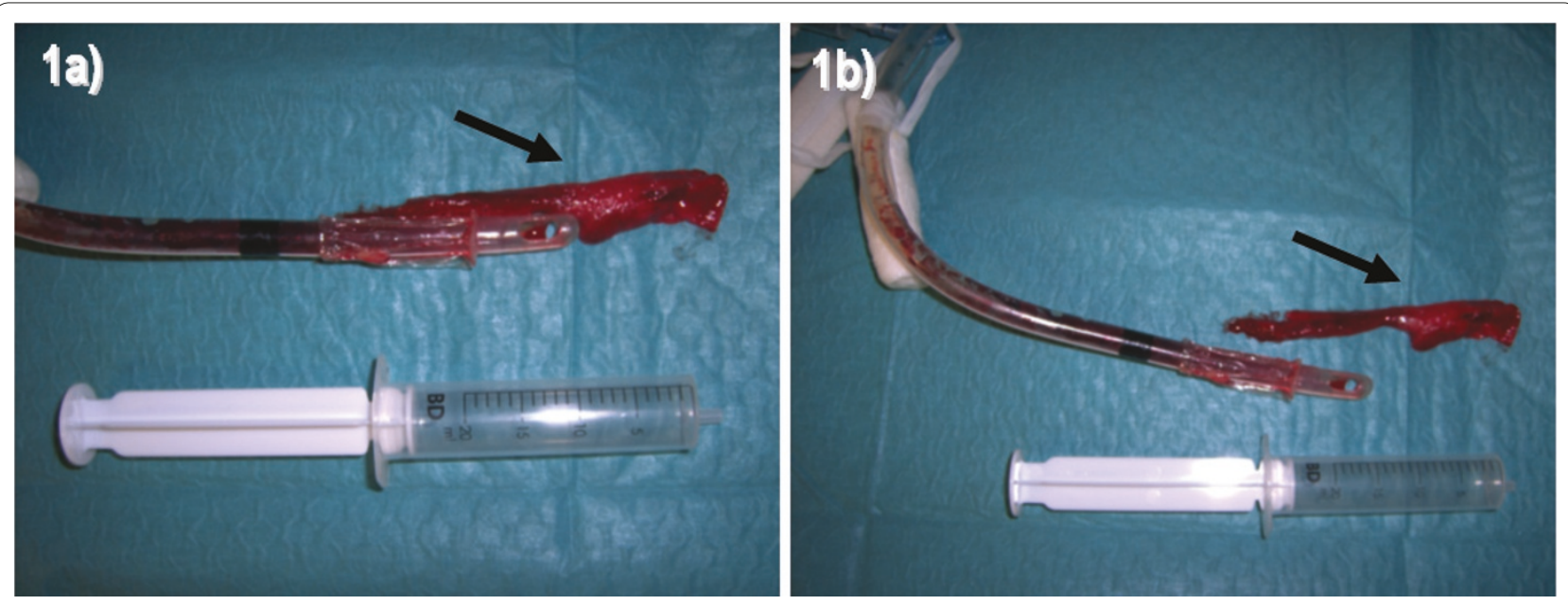

Figure 1. A bronchial thrombus critically obstructed the airways in a long-term ventilated patient under recombinant factor VIla and factor VIII therapy. (a) The thrombus (arrow) was still attached to the tip of the removed endotracheal tube. (b) The detached thrombus (arrow) was approximately $20 \times 70 \mathrm{~mm}$. With its solid consistency, it occluded the tip of the endotracheal tube and the airways like a cork.

Recommendations on the use of recombinant activated factor VII as an adjunctive treatment for massive bleeding--a European perspective. Crit Care 2006, 10:R120.

\section{doi:10.1186/cc10041}

Cite this article as: Lauer S, et al.: Recombinant factor VIla and factor VIII treatment for acquired factor VIII deficiency: a case of repeated thrombotic endotracheal occlusion in a mechanically ventilated patient. Critical Care 2011, 15:407. 